\title{
Immunocytochemical detection of XIAP in body cavity effusions and washes
}

\author{
Maoxin Wu, Songyang Yuan, Arnold H Szporn, Li Gan, Viktor Shtilbans and \\ David E Burstein
}

Department of Pathology, Mount Sinai School of Medicine, New York, NY, USA

\begin{abstract}
Body cavity effusions may be the first manifestation of malignancy or of recurrence or relapse. We surveyed effusions and washes for expression of X-linked inhibitor of apoptosis (XIAP), a potent constituent of the inhibitor of apoptosis (IAP) family of proteins. IAPs prevent apoptosis by blocking the activation of caspases, thereby preventing caspase-mediated cell degradation. Elevated expression of XIAP could be an underpinning of relapse and/or resistance to apoptosis-inducing cancer therapy. We performed an immunocytochemical survey of XIAP expression in cell blocks from benign and malignant body cavity effusions and washes. In all, 116 alcohol-fixed, formalin postfixed paraffin-embedded cell block specimens from 82 pleural effusions, 22 ascites, $11 \mathrm{pelvic/peritoneal} \mathrm{washes} \mathrm{and} \mathrm{one} \mathrm{pericardial} \mathrm{effusion} \mathrm{were} \mathrm{evaluated} \mathrm{immunocytochemically} \mathrm{with}$ monoclonal anti-XIAP (\#610763, BD Biosciences, San Jose, USA) 1:250, $4^{\circ} \mathrm{C} \times 72 \mathrm{~h}$, and developed using EnVision-Plus reagents (Dako) and diaminobenzidine as chromagen. Particulate cytoplasmic staining was considered positive. The prevalence of staining for specific malignancies varied with the tissue of origin as follows: ovarian $(13 / 13,100 \%)$; lung $(9 / 11,82 \%)$, breast $(6 / 13,46 \%)$; gastric $(4 / 7,57 \%)$, colon $(0 / 4,0 \%)$, pancreas $(2 / 3,67 \%)$, gallbladder $(1 / 1,100 \%)$, fallopian tube $(1 / 3,33 \%)$, endometrial $(6 / 7,86 \%)$, mesothelioma $(4 / 5,80 \%)$, carcinoma of unknown primary $(5 / 5,100 \%)$ and hematopoietic malignancies $(3 / 9,33 \%)$. Overall, 54 out of 81 $(67 \%)$ malignant effusions displayed XIAP positivity. Benign effusions $(n=35)$ were virtually XIAP-negative except for two cases $(6 \%)$ in which histiocytes showed moderate staining. Weak nonspecific staining was sometimes noted in inflammatory cells or histiocytes. XIAP immunostaining, when strong, allows for ready distinction of malignant from benign and reactive cell populations. Strong XIAP staining was most prevalent in ovarian carcinomas and less prevalent in mammary carcinomas. The degree of XIAP staining of tumor cells may be a means of identifying the most therapy-resistant cases (ie, those with strong XIAP expression), and allow additional triaging to XIAP-blocking drugs presently being developed and clinically tested.
\end{abstract}

Modern Pathology (2005) 18, 1618-1622. doi:10.1038/modpathol.3800478; published online 19 August 2005

Keywords: ascites; pleural effusion; pericardial effusion; XIAP; apoptosis; cancer therapy

Body cavity effusions may be the presenting sign of malignancy or the first manifestation of recurrence and relapse. Tumor spread via survival and proliferation of tumor cells in body cavity fluid is an important route of metastasis in malignancies such as ovarian carcinomas, and a frequent cause of morbidity in many malignancies. A more complete understanding of the factors and pathways that permit survival and proliferation of malignant cells suspended in body cavity fluids could lead to more effective intervention and prevention of this route of tumor spread.

Correspondence: Dr M Wu, MD, PhD and Dr DE Burstein, MD, Department of Pathology, Mount Sinai School of Medicine, 1 Gustave Levy Place, New York, NY 10029, USA.

E-mails: Maoxin.Wu@msnyuhealth.org;

David.Burstein@msnyuhealth.org

Received 4 May 2005; revised and accepted 12 July 2005; published online 19 August 2005
In general, tumor cells differ from their normal cells of origin in blunting of the apoptotic response to cell stressors. ${ }^{1}$ Metabolic adaptations that enhance survival such as induction of hypoxiaresponsive genes and activation of autocrine growth-promoting pathways also occur frequently in cancer cells, and have been demonstrated in malignant effusions in particular. ${ }^{2,3}$ An inter-related group of eight proteins, designated inhibitors of apoptosis (IAPs), ${ }^{4}$ XIAP(X-linked inhibitor of apoptosis), ILP-2, c-IAP1, c-IAP2, ML-IAP (livin), NAIP, survivin (TIAP), apollon (Bruce) are emerging as clinically important promoters of tumor cell survival and as suppressors of tumor cell response to apoptosis-inducing cancer therapies. IAPs prevent apoptosis by blocking the activation of specific caspases, thereby preventing caspase-mediated cell degradation. The ability of XIAP, reportedly the most potent of the IAPs, to inhibit caspases 3, 7 and 
9 places it in a critical position to block both intrinsic and extrinsic apoptotic pathways. ${ }^{5}$ Elevated expression of XIAP could thus be an underpinning of both enhanced survivability of cancer cells and of resistance to apoptosis-inducing cancer therapy, leading to clinical relapse. Conversely, pharmacologic suppression of XIAP could restore sensitivity to tumor cells with XIAP-mediated resistance to tumor therapy. ${ }^{6}$ Two compounds with XIAP-inhibitory activity, phenoxodiol and flavopiridol, are already in clinical trials. ${ }^{7-10}$

We describe below an immunocytochemical survey of XIAP expression in benign and malignant effusions and washes, and demonstrate XIAP positivity in a significant proportion of malignant effusions. The clinical and biologic implications are discussed.

\section{Methods}

Sections $(4 \mu \mathrm{m})$ from 81 malignant and 35 benign ethanol-fixed, formalin postfixed paraffin-embedded cell block specimens from 82 pleural effusions, 22 ascites, 11 pelvic or peritoneal washes and one pericardial effusion were deparaffinized, exposed to $3 \%$ hydrogen peroxide to block endogenous peroxidase activity, incubated for antigen retrieval with $0.01 \mathrm{M}$ citric acid at $\mathrm{pH} 6.0$ and at $100^{\circ} \mathrm{C} \times 5 \mathrm{~min}$, followed by cooling for $20 \mathrm{~min}$, then incubated with monoclonal anti-XIAP (\#610763, BD Biosciences, San Jose, USA) diluted 1:250 in PBS with $5 \%$ albumin and $5 \%$ goat serum at $4{ }^{\circ} \mathrm{C} \times 72 \mathrm{~h}$, and developed using EnVision-Plus reagents (Dako) and diaminobenzidine as chromagen. Only distinctly particulate cytoplasmic staining was scored as positive and graded as $1+$ (weak), $2+$ (moder- ate), and $3-4+$ (strong). Primary sources of tumors were as follows: 13 ovary; 11 lung; 13 breast; 7 gastric; 7 endometrial; 5 mesothelial; 5 carcinomas of unknown primary; 3 fallopian tube; 4 colon; 3 pancreas; 1 gall bladder; 9 hematopoietic.

\section{Results}

The summary data are presented in Table 1.

Benign effusions $(n=35)$ were XIAP-negative (Figure 1a), or showed nonspecific or focal-weak staining of mesothelial cells and macrophages in both noninflammatory specimens and specimens with acute or chronic inflammatory cells. Neutrophils and lymphocytes were characteristically negative. In rare benign effusions (two out of 35), staining of moderate intensity in a significant number of cells, mostly histiocytes (Figure 1b), possibly artifactual, was noted.

Unlike staining in benign effusions, XIAP positivity was frequently detected in malignant effusions. Positive staining was manifested by granular cytoplasmic staining that readily distinguished malignant from nonmalignant cell populations (Figure 2a-d). Staining positivity varied among different tumor types, with high prevalence in carcinomas of ovarian $(13 / 13,100 \%)$, pulmonary $(9 / 11,82 \%)$ and endometrial origin $(6 / 7,86 \%)$, and mesotheliomas $(4 / 5,80 \%)$. Positivity ranged from strong and/or diffuse to weak and/or focal. In contrast, XIAP staining was less prevalent in effusions of metastatic mammary carcinoma (6/13, $46 \%)$. Overall, 54 out of 81 malignant effusions $(67 \%)$ displayed XIAP positivity.

Among tumor types found less frequently in effusions or peritoneal washes, positive staining

Table 1 Summary of XIAP staining in malignant and benign body cavity effusions and washes

\begin{tabular}{|c|c|c|c|c|c|}
\hline $\begin{array}{l}\text { Case type } \\
\text { (primary) }\end{array}$ & $\begin{array}{l}\text { No. of cases } \\
\text { (type of fluid) }\end{array}$ & $\begin{array}{l}\text { Positive } \\
\text { for XIAP }\end{array}$ & $\begin{array}{l}\text { Staining intensity } \\
\text { (o to }++++)\end{array}$ & $\begin{array}{l}\% \text { of XIAP + } \\
\text { cases }\end{array}$ & Comments \\
\hline Ovarian & $13(6 \mathrm{P}+6 \mathrm{~A}+1 \mathrm{PW})$ & 13 & + to ++++ & 100 & Mostly strong \\
\hline Lung & $11(10 \mathrm{P}+1 \mathrm{PC})$ & 9 & + to ++++ & 82 & Mostly strong in positive cells \\
\hline Breast & $13(11 \mathrm{P}+2 \mathrm{~A})$ & 6 & + to +++ & 46 & Not as strong as ovarian/lung \\
\hline Gastric & $7(5 \mathrm{P}+2 \mathrm{~A})$ & 4 & + to +++ & 57 & Two cases from same patient \\
\hline Colon & $4(1 \mathrm{P}+2 \mathrm{~A}+1 \mathrm{PW})$ & 0 & - & 0 & \\
\hline Pancreas & $3(2 \mathrm{P}+1 \mathrm{~A})$ & 2 & + to +++ & 67 & \\
\hline Gall bladder & $1(1 \mathrm{~A})$ & 1 & ++ to +++ & 100 & \\
\hline Fallopian tube & $3(1 \mathrm{~A}+2 \mathrm{PW})$ & 1 & + to +++ & 33 & \\
\hline Endometrial & $7(1 \mathrm{P}+1 \mathrm{~A}+5 \mathrm{PW})$ & 6 & + to ++++ & 86 & \\
\hline Mesothelioma & $5(4 P+1 A)$ & 4 & + to ++++ & 80 & \\
\hline Carcinoma NOS & $5(5 P)$ & 5 & +++ to ++++ & 100 & \\
\hline $\begin{array}{l}\text { Hematopoietic } \\
\text { malignancies }\end{array}$ & $9(9 \mathrm{P})$ & 3 & + to ++ & 33 & $\begin{array}{l}\text { Weak stain with Golgi-like } \\
\text { distribution seen in rare } \\
\text { cells of MM and two NHL cases }\end{array}$ \\
\hline Negative & $35(28 \mathrm{P}+5 \mathrm{~A}+2 \mathrm{PW})$ & 2 & + to ++ & 6 & $\begin{array}{l}\text { Usually negative; histiocytes } \\
\text { positive in rare cases }\end{array}$ \\
\hline Summary & $\begin{array}{l}\text { Total }=116(81+35) \\
(82 \mathrm{P}+22 \mathrm{~A}+11 \mathrm{PW}+1 \mathrm{PC})\end{array}$ & 56 & & & $\begin{array}{l}66.7 \%(54 / 81) \text { malignant cases } \\
\text { are positive for XIAP }\end{array}$ \\
\hline
\end{tabular}

$\mathrm{P}=$ pleural fluid; $\mathrm{A}=$ ascites; $\mathrm{PW}=$ pelvic washing; $\mathrm{PC}=$ pericardial. 


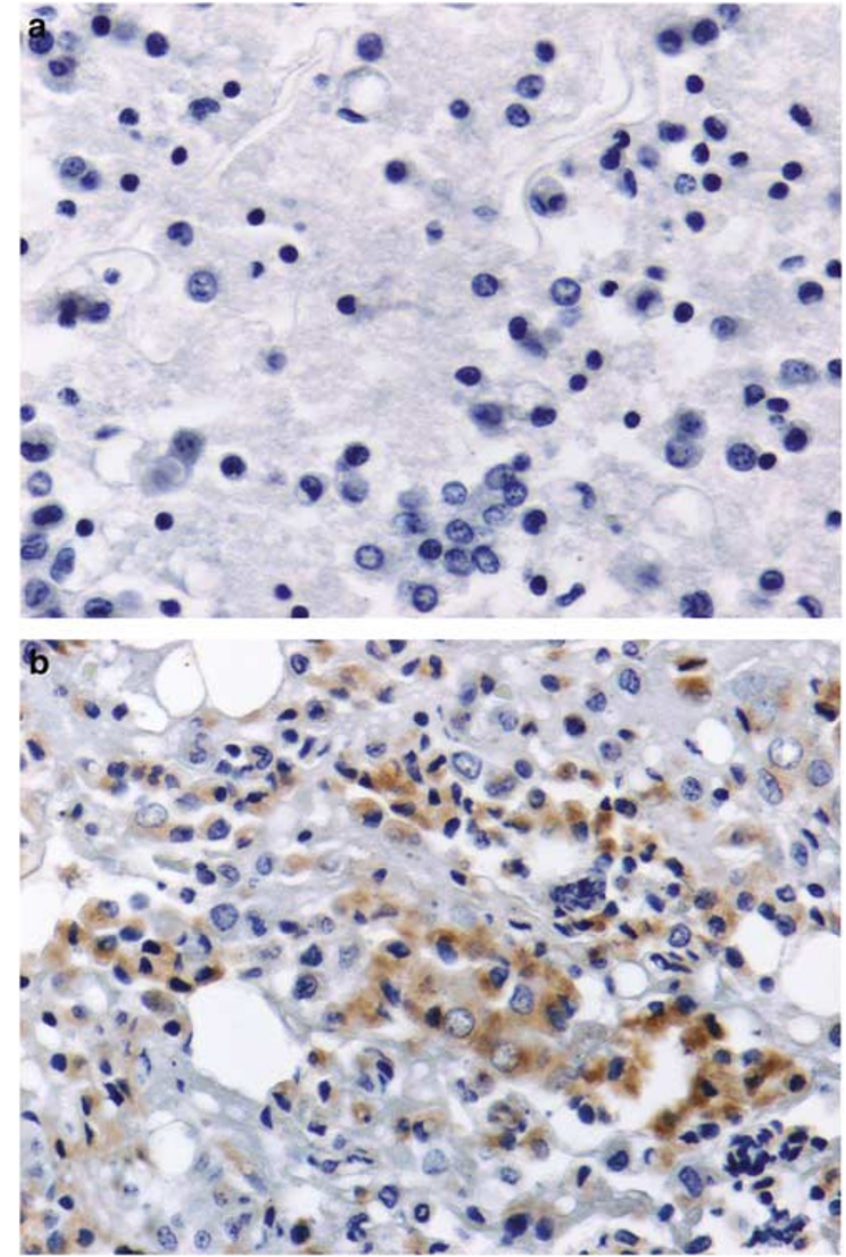

Figure 1 XIAP immunstaining of benign effusions. (a) Absence of staining of mesothelial cells, histiocytes and inflammatory cells, benign effusion. (b) Moderate positive staining in significant number of histiocytes in a rare benign effusion.

was noted in two out of three pancreatic adenocarcinomas; one of the three fallopian tube carcinomas; one of one gallbladder carcinoma and no colon carcinomas $(0 / 4)$.

Nine hematopoietic malignancies (six non-Hodgkin's lymphomas, one CLL, one AML, and one multiple myeloma) were examined. The myeloma and two of the non-Hodgkin's lymphomas displayed a Golgi-like staining distribution in scattered to rare cells, the significance of which is uncertain; several tissue sections of myeloma were nonstaining, although a subset of lymphoma tissue sections was found to be XIAP-positive (unpublished data). A case of primary effusion lymphoma was nonstaining.

Five of the five carcinomas of unknown primary, including one with signet-ring morphology, were strongly and diffusely positive. Seven gastric carcinoma-induced effusions were examined, two from the same patient. This patient's earlier specimen was XIAP-negative; however, the repeat specimen, taken 50 days later, was XIAP-positive. Of note, the earlier specimen showed a significantly higher percentage $(17 \%)$ of apoptotic-like degenerated cells compared to the later specimen (7\%), possibly related to upregulated expression and apoptoticinhibitory activity of XIAP (Figure 3a and b). A metastatic gastric carcinoma of intestinal type showed only equivocal-to-weak staining. Overall, four of the seven gastric carcinoma specimens were positively stained.

Although the vast majority of benign or reactive mesothelial cells were nonstaining or displayed weak or nonspecific staining, four of five malignant mesothelioma specimens were positively stained, suggesting that strong XIAP staining in a cell population with mesothelial differentiation may signify malignancy.

\section{Discussion}

One feature that distinguishes many cancer cells from their normal counterparts is the aberrant activation of pathways that promote survival and/ or suppress apoptosis. Normal mesothelial cells that exfoliate into body cavities presumably survive only transiently and fail to proliferate, analogous to the inability of nontransformed cells suspended in soft agar in vitro to achieve anchorage-independent growth. In contrast, tumor cells of many types adapt well to suspension in body cavity effusions, and survive as well as proliferate.

Contrasting with tumor cell growth in tissue, survival and proliferation of malignant cells in body cavity effusions occur despite the absence of an adjacent capillary oxygen source; absence of adhesion to stromal or endothelial cells or to acellular matrix or adhesion molecules, all of which may have paracrine effects on tumor cells; the frequent presence of low oxygen tension, high lactate levels, low glucose and acidic $\mathrm{pH},{ }^{11,12}$ all environmental stressors that might minimize survival and proliferation and/or induce cell death in nontransformed cells. Given the considerable differences in microenvironment from tumor cells growing in tissue phase, it is not surprising that tumor cells from effusions may differ biologically from cells in the associated solid tumor. ${ }^{3}$

We have previously demonstrated one presumptive survival-promoting adaptation of malignant cells suspended in effusions-aberrant elevation of GLUT1, a hypoxia-inducible glucose transporter that occurs in a significant percentage of malignant effusions. ${ }^{2}$ GLUT1 elevation may be an adaptative response of malignancy to low oxygen tension, possibly leading to increased generation of ATP via glycolysis, a well-known feature of malignancy first reported by Warburg ${ }^{13}$ in the 1920s.

Other biologic changes that have been proposed as permitting the selective survival of malignant cells in effusions include induction of autocrine pathways. $^{3}$ 

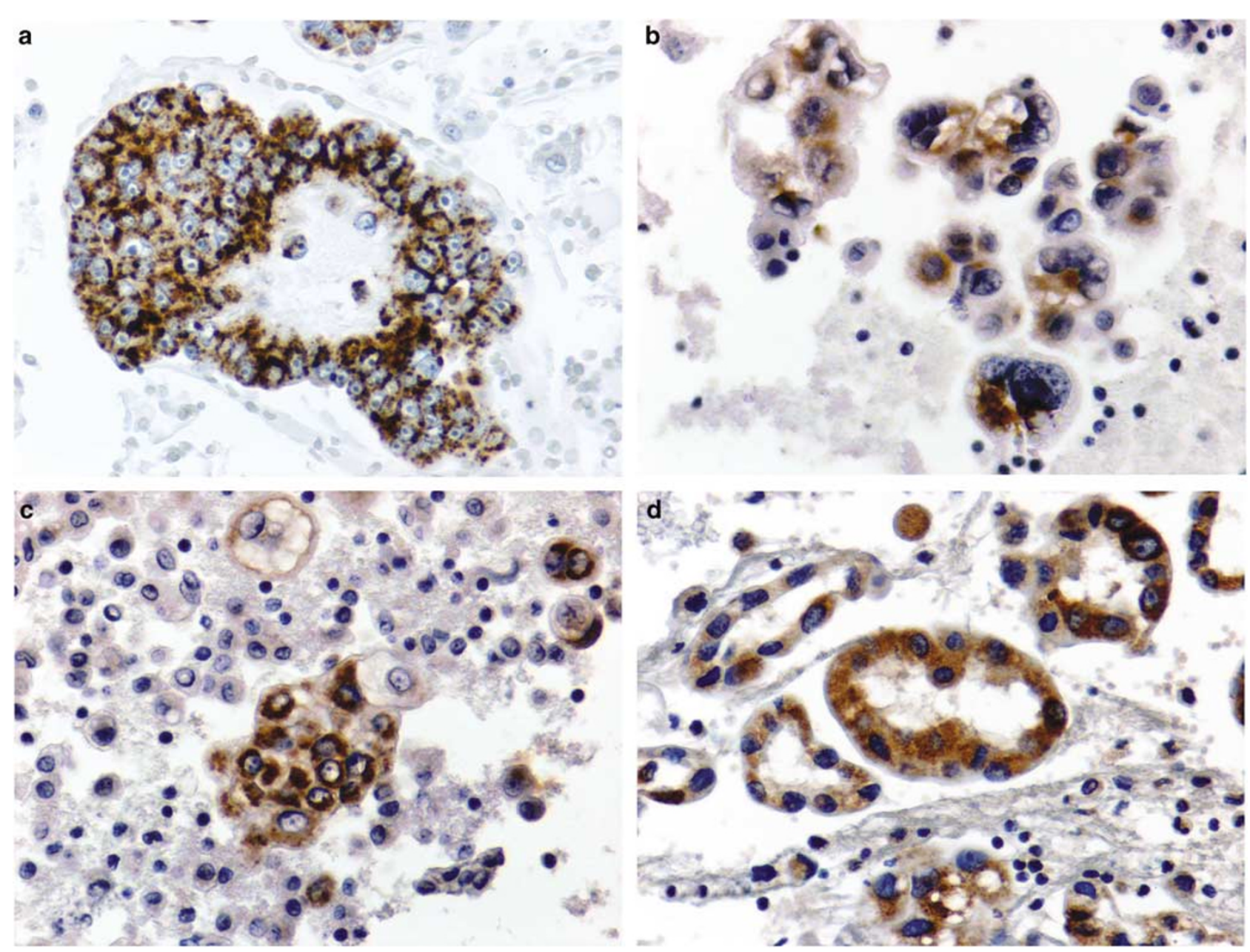

Figure 2 XIAP immunostaining of malignant effusions: (a) ovarian adenocarcinoma (note nonstaining of surrounding benign mesothelial cells or histiocytes), (b) lung carcinoma, (c) mesothelioma, (d) breast carcinoma.

The present findings suggest that high expression of XIAP is another common feature of carcinoma cells in effusions that may play a role in promoting tumor cell survival. Only a few studies have provided evidence for reduced apoptosis in malignant effusions. Significantly enhanced levels of soluble Fas, a molecule that inhibits Fas receptormediated apoptosis, were reported in malignant effusions, compared to levels in tuberculous effusions and transudates. Lung cancer cell lines were also shown to synthesize soluble Fas. ${ }^{14}$ Conversely, an activator of death-receptor-mediated apoptosis, tumor-necrosis factor-related apoptosis-inducing ligand (TRAIL), was synthesized by macrophages from various malignant effusions; levels were reported to be higher in effusions with fewer tumor cells. ${ }^{15}$

Among tumors commonly associated with development of malignant effusions, strong XIAP staining was most prevalent in ovarian, pulmonary and endometrial carcinomas, and less prevalent in mammary carcinomas. A significant degree of XIAP immunostaining allows for ready distinction of malignant from benign and reactive cell populations in a high percentage of cases, the only exceptions being rare benign specimens in which diffuse, possibly artifactual positivity of various cell types was noted. Of interest, the consistently low expression of XIAP in benign mesothelial cells contrasts with XIAP positivity noted in four of five malignant mesothelioma specimens studied, suggesting a specific diagnostic application.

Effusions caused by malignant hematopoietic cells showed variable degrees of positivity, consistent with positivity in a subset of non-Hodgkin's lymphomas as well as positivity in Reed-Sternberg cells in Hodgkin's disease ${ }^{16}$ our unpublished data. Unexplained is the nature of positive XIAP staining in a Golgi-like distribution.

Since benign cells are nonstaining in the great majority of cases, XIAP immunostaining may be an acceptable means of establishing or confirming the malignant nature of suspicious cell populations in effusion or wash samples.

Elevated XIAP expression has been linked to resistance to therapy in diffuse B-cell lymphoma ${ }^{17}$ 

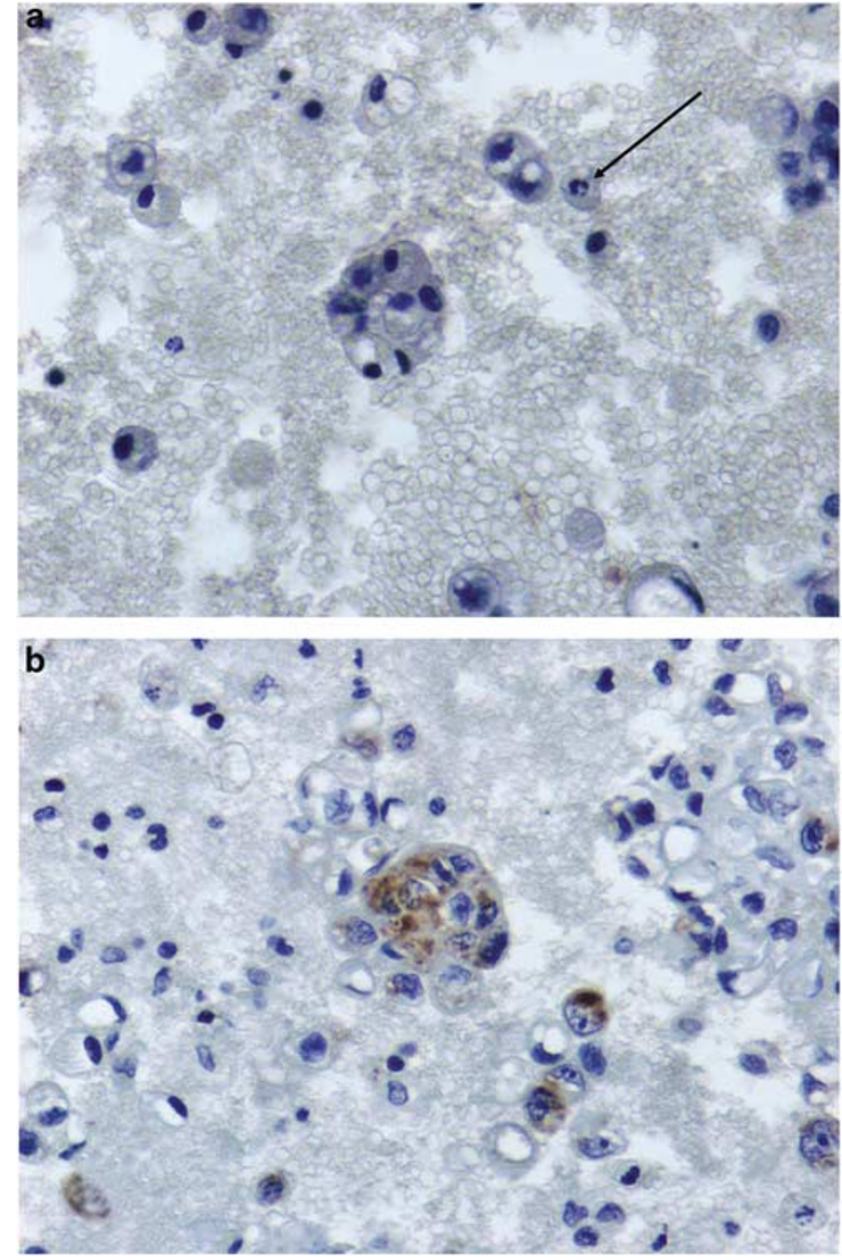

Figure 3 XIAP in sequential ascites samples of metastatic gastric carcinoma. (a) XIAP is negative in tumor cells. Note apoptoticappearing cells (arrow). (b) XIAP is positive in ascites 50 days later. Specimen had fewer apoptotic-appearing cells than in (a).

and $\mathrm{AML}^{18}$ and to more aggressive clinical behavior or higher tumor grade in renal, ${ }^{19}$ uterine cervical squamous and endometrial carcinoma (unpublished data). The degree of XIAP staining of tumor cells may be a means of identifying effusions, and possibly their associated solid tumors, most resistant to apoptosis-inducing cancer therapies (ie, those with strong XIAP expression), and in the near future allow additional triaging to XIAP-blocking drugs presently being developed and clinically tested..$^{9,10}$

\section{Acknowledgement}

This study is supported by a generous bequest to DB from the Estate of Hilda Leveen.

\section{References}

1 Hanahan D, Weinberg RA. The hallmarks of cancer. Cell 2000;100:57-70.

2 Burstein DE, Reder I, Weiser K, et al. GLUT1 glucose transporter: a highly sensitive marker of malignancy in body cavity effusions. Mod Pathol 1998;11:392-396.

3 Davidson B. Malignant effusions: from diagnosis to biology. Diagnostic Cytopathol 2004;31:246-254.

4 Salvesen GS, Duckett CS. IAP proteins: blocking the road to death's door. Nat Rev Mol Cell Biol 2002;3: 401-410.

5 Holcik M, Korneluk RG. XIAP, the guardian angel. Nat Rev Mol Cell Biol 2001;2:550-556.

6 Sapi E, Alvero AB, Chen W, et al. Resistance of ovarian carcinoma cells to docetaxel is XIAP dependent and reversible by phenoxodiol. Oncol Res 2004;14:567-578.

7 Kamsteeg M, Rutherford T, Sapi E, et al. Phenoxodiolan isoflavone analog-induces apoptosis in chemoresistant ovarian cancer cells. Oncogene 2003;22:2611-2620.

8 Rosato RR, Dai Y, Almenara JA, et al. Potent antileukemic interactions between flavopiridol and TRAIL/ Apo2L involve flavopiridol-mediated XIAP downregulation. Leukemia 2004;18:1780-1788.

9 Omalley DM, Mor G, Makkenchery A, et al. Phase Ib/II study of phenoxodiol in patients with recurrent ovarian, fallopian and primary peritoneal cancer that is resistant to second line chemotherapy. Presented at the 2004 Annual Meeting on Women's Cancer, Society of Gynecologic Oncologists, February 2004, San Diego, California.

10 Shapiro GI. Preclinical and clinical development of the cyclin-dependent kinase inhibitor flavopiridol. Clin Cancer Res 2004;10:4270s-4275s.

11 Sahn SA, Good JT. Pleural fluid $\mathrm{pH}$ in malignant effusions. Ann Intern Med 1988;108:345-349.

12 Good JT, Taryle DA, Sahn SA. Pathogenesis of low glucose/low $\mathrm{pH}$ malignant effusions. Ann Rev Respir Dis 1985;131:737-741.

13 Warburg O. On the origin of cancer cells. Science 1956;123:309-314.

14 Mitani K, Nishioka Y, Yamabe K, et al. Soluble Fas in malignant pleural effusion and its expression in lung cancer cells. Cancer Sci 2003;94:302-307.

15 Herbeuval JP, Lambert C, Sabido O, et al. Macrophages from cancer patients: analysis of TRAIL, TRAIL receptors, and colon tumor cell apoptosis. J Natl Cancer Inst 2003;95:611-621.

16 Kashkar H, Haefs C, Shin H, et al. XIAP-mediated caspase inhibition in Hodgkin's lymphoma-derived B cells. J Exp Med 2003;198:341-347.

17 Muris JJ, Cillessen SA, Vos W, et al. Immunohistochemical profiling of caspase signaling pathways predicts clinical response to chemotherapy in primary nodal diffuse large B-cell lymphomas. Blood 2004, Dec 2 [Epub ahead of print].

18 Tamm I, Richter S, Scholz F, et al. XIAP expression correlates with monocytic differentiation in adult de novo AML: impact on prognosis. Hematol J 2004;5: 489-495.

19 Ramp U, Krieg T, Caliskan E, et al. XIAP expression is an independent prognostic marker in clear-cell renal carcinomas. Hum Pathol 2004;35:1022-1028. 